\title{
Kähler decomposition of 4-manifolds
}

\author{
R İNANÇ BAYKUR
}

\begin{abstract}
In this article we show that every closed oriented smooth 4-manifold can be decomposed into two codimension zero submanifolds (one with reversed orientation) so that both pieces are exact Kähler manifolds with strictly pseudoconvex boundaries and that induced contact structures on the common boundary are isotopic. Meanwhile, matching pairs of Lefschetz fibrations with bounded fibers are offered as the geometric counterpart of these structures. We also provide a simple topological proof of the existence of folded symplectic forms on 4-manifolds.
\end{abstract}

57R17, 57M50; 57N13

\section{Introduction}

One possible strategy for understanding oriented smooth 4-manifolds is to break them up into more tractable classes of manifolds in a controlled manner. Situated in the intersection of complex, symplectic and Riemannian geometries, Kähler manifolds are the best known candidates to be pieces of such a decomposition. The main goal of this article is to show that this can be achieved for any closed oriented smooth 4-manifold $X$. We decompose $X$ into two exact Kähler manifolds with strictly pseudoconvex boundaries, up to orientation, such that contact structures on the common boundary induced by the maximal complex distributions are isotopic.

The decomposition gives rise to a globally defined 2-form on $X$, which we will call a (nicely) folded Kähler structure, and it belongs to a larger family of 2-forms: folded symplectic structures. Cannas da Silva showed in [5] that any closed smooth oriented 4-manifold can be equipped with a folded symplectic form, by using a version of the h-principle defined for folding maps by Eliashberg. In Section 3, we introduce a way to construct some simple examples of folded symplectic 4-manifolds. Afterwards we reprove the existence fact by constructing a folded symplectic form $\omega$ for a given handlebody decomposition of $X$, essentially by means of simple Kirby calculus and contact topology (Theorem 4.1). The main ingredient there will be achiral Lefschetz fibrations, and recent work of Etnyre and Fuller [12] will play a key role in our construction. 
Next, we switch gears, and using several results on compact Stein surfaces and Lefschetz fibrations with bounded fibers (mainly Harer [20], Eliashberg [8], Gompf [17], LoiPiergallini [23], and Akbulut-Ozbagci [2]), we prove the aforementioned decomposition theorem (Theorem 5.2). In fact we obtain a stronger result, as the pieces of this decomposition are actually Stein manifolds with strictly pseudoconvex boundaries. It was first shown by Akbulut and Matveyev in [1] that a closed oriented smooth 4manifold $X$ could always be decomposed into Stein pieces, but there was no particular information one could use to argue for matching the induced contact structures on the separating hypersurface. Our proof follows an alternative way via open book decompositions, and we conclude that the Stein structures can be chosen to agree on the common contact boundary.

In the last section, we introduce folded Kähler structures, and discuss some properties they enjoy, after showing that all closed oriented smooth 4-manifolds admit them (Theorem 6.2). This improves the folded symplectic existence result, and indeed both structures we construct are shown to be equivalent on the symplectic level. The collection of these discussions leads us to define folded Lefschetz fibrations, roughly speaking, pairs of positive and negative Lefschetz fibrations over disks with bounded fibers which agree on the common boundary through induced open book decompositions. We prove that any nicely folded Kähler 4-manifold, possibly after an orientation preserving diffeomorphism, admits compatible folded Lefschetz fibrations (Proposition 6.6).

Acknowledgments The author would like to thank Ron Fintushel for his support and for helpful conversations. The author would also like to thank Burak Ozbagci for commenting on the draft of this paper. The author was partially supported by NSF Grant DMS0305818.

\section{Preliminaries}

\subsection{Lefschetz fibrations}

All manifolds and maps in this article are assumed to be smooth. A Lefschetz fibration on an oriented 4-manifold $X$, possibly with boundary, is a map $f: X \rightarrow \Sigma$, where $\Sigma$ is a compact oriented surface, such that each critical point of $f$ lies in the interior of $X$ and has a local model $f\left(z_{1}, z_{2}\right)=z_{1} z_{2}$, given by orientation preserving charts both on $X$ and $\Sigma$. These singularities are obtained by attaching 2-handles to regular fibers with framing -1 with respect to the framing induced by the fiber. We will refer to these $2-$ handles as positive Lefschetz handles. An achiral Lefschetz fibration is defined the same 
way, except that the given charts around critical points are allowed to reverse orientation. In other words, the 2 -handles can be glued with framing +1 with respect to fiber framing, too. Also recall that a Lefschetz pencil is a map $f: X \backslash\left\{b_{1}, \ldots, b_{k}\right\} \rightarrow S^{2}$, such that around any base point $b_{i}$ it has a local model $f\left(z_{1}, z_{2}\right)=z_{1} / z_{2}$, preserving the orientations, and that $f$ is a Lefschetz fibration elsewhere. An achiral Lefschetz pencil is then defined by allowing orientation reversing charts around the base points as well. Critical points or base points with orientation reversing charts are called negative critical points or negative base points, whereas the other critical points or base points are positive. For a detailed treatment of this topic and proofs of some facts quoted below, the reader is advised to turn to Gompf and Stipsicz [18].

A Lefschetz fibration is said to be allowable if all its vanishing cycles are homologically nontrivial in the fiber. Particularly, we will be interested in allowable Lefschetz fibrations over $D^{2}$ with bounded fibers. In the literature, this type of Lefschetz fibration having only positive critical points is called a $P A L F$. Similarly, when the critical points are instead all negative, we will call the fibration a $N A L F$.

Given a compact oriented genus $g$ surface $F$ with $m$ boundary components and $r$ marked points on it, the mapping class group $\Gamma_{g, m}^{r}$ is defined as the group of orientation preserving self-diffeomorphisms of $F$ fixing marked points and $\partial F$ pointwise, modulo isotopies of $F$ fixing marked points and $\partial F$ pointwise. It can be shown that $\Gamma_{g, m}^{r}$ is generated by positive (right-handed) and negative (left-handed) Dehn twists. Importantly, isotopy type of a surface bundle over $S^{1}$ with fiber closed oriented surface $F$ is determined by the return map of a flow transverse to the fibers, which can be identified with an element $\mu \in \Gamma_{g}$, called monodromy of this fibration.

Let $f: X \rightarrow D^{2}$ be an achiral Lefschetz fibration, where the regular fiber $F$ is an oriented genus $g$ surface with $m$ boundary components, and suppose all critical points of the fibration lie on various fibers. Select a regular value $p$ in the interior of $D^{2}$, an identification of $f^{-1}(p) \cong F$, and a collection of $\operatorname{arcs} s_{1}, \ldots, s_{k}$ in the interior of $D^{2}$ with each $s_{i}$ connecting $p$ to a distinct critical value, and all disjoint except at $p$. We index the critical values as well, so that each arc $s_{i}$ is connected to a critical value $q_{i}$ and that they appear in a counterclockwise order around the point $p$. Now if we take a regular neighborhood of each arc away from remaining critical points and consider the union of these, we obtain a disk $V$ and an $F$-bundle over $\partial V=S^{1}$. The monodromy of this fibration is an element $\mu \in \Gamma_{g, m}$, which is called the global monodromy of the achiral Lefschetz fibration $f$. It is well-known that this data gives a handlebody description of $X$, and vice versa. We call the ordered set of $\operatorname{arcs}\left\{s_{1}, \ldots, s_{k}\right\}$ a representation of the achiral Lefschetz fibration $f$.

Next is a standard fact which was first observed by Harer: 
Theorem 2.1 (Harer [20]) Let $X$ be a 4-manifold with boundary. Then $X$ admits an achiral Lefschetz fibration over $D^{2}$ with bounded fibers if and only if it admits a handlebody decomposition with no handle of index greater than two.

\subsection{Open book decompositions}

An open book decomposition of a 3 -manifold $M$ is a pair $(B, f)$ where $B$ is an oriented link in $M$, called the binding, and $f: M \backslash B \rightarrow S^{1}$ is a fibration such that $f^{-1}(t)$ is the interior of a compact oriented surface $F_{t} \subset M$ and $\partial F_{t}=B$ for all $t \in S^{1}$. The surface $F=F_{t}$, for any $t$, is called the page of the open book. The monodromy of an open book is given by the return map of a flow transverse to the pages and meridional near the binding, which is an element $\mu \in \Gamma_{g, m}$, where $g$ is the genus of the page $F$, and $m$ is the number of components of $B=\partial F$.

Suppose we have an achiral Lefschetz fibration $f: X \rightarrow D^{2}$ with bounded regular fiber $F$, and let $p$ be a regular value in the interior of the base $D^{2}$. Composing $f$ with the radial projection $D^{2} \backslash\{p\} \rightarrow \partial D^{2}$ we obtain an open book decomposition on $\partial X$ with binding $\partial f^{-1}(p)$. Identifying $f^{-1}(p) \cong F$, we can write $\partial X=(\partial F \times$ $\left.D^{2}\right) \cup f^{-1}\left(\partial D^{2}\right)$. Thus we view $\partial F \times D^{2}$ as the tubular neighborhood of the binding

$B=\partial f^{-1}(p)$, and the fibers over $\partial D^{2}$ as its truncated pages. The monodromy of this open book is prescribed by that of the achiral fibration [20]. In this case, we say the open book $\left(B,\left.f\right|_{\partial X \backslash B}\right)$ bounds or is induced by the achiral Lefschetz fibration $f: X \rightarrow D^{2}$. Recalling that any closed oriented 3-manifold can be bounded by a 4-manifold with only $0-, 1-$ and $2-$ handles, it is fairly easy to see that any open book decomposition bounds such an achiral Lefschetz fibration over a disk.

We would like to describe an elementary modification of these structures: Let $f: X \rightarrow$ $D^{2}$ be an achiral Lefschetz fibration with bounded regular fiber $F$. Attach a 1 -handle to $\partial F$ to obtain $F^{\prime}$, and then attach a positive (resp. negative) Lefschetz 2-handle along an embedded loop in $F^{\prime}$ that goes over the new 1-handle exactly once. This is called a positive stabilization (resp. negative stabilization) of $f$. A positive (resp. negative) Lefschetz handle is attached with framing -1 (resp. +1$)$ with respect to the fiber, and thus it introduces a positive (resp. negative) Dehn twist on $F^{\prime}$. If the focus is on the 3-manifold, one can totally forget the bounding 4-manifold and view all the handle attachments in the 3-manifold. Either way, stabilizations correspond to adding canceling handle pairs, so diffeomorphism types of the underlying manifolds do not change, whereas the achiral Lefschetz fibration and the open book decomposition change in the obvious way. It turns out that stabilizations preserve more than the underlying topology, as we will discuss shortly. 


\subsection{Contact structures and compatibility}

A 1 -form $\alpha \in \Omega^{1}(M)$ on a $(2 n-1)$-dimensional oriented manifold $M$ is called a contact form if it satisfies $\alpha \wedge(d \alpha)^{n-1} \neq 0$. An oriented contact structure on $M$ is then a hyperplane field $\xi$ which can be globally written as kernel of a contact 1 -form $\alpha$. In dimension three, this is equivalent to asking that $d \alpha$ be nondegenerate on the plane field $\xi$.

A contact structure $\xi$ on a 3 -manifold $M$ is said to be supported by an open book $(B, f)$ if $\xi$ is isotopic to a contact structure given by a 1 -form $\alpha$ satisfying $\alpha>0$ on positively oriented tangents to $B$ and $d \alpha$ is a positive volume form on every page. When this holds, we say that the open book $(B, f)$ is compatible with the contact structure $\xi$ on $M$.

Improving results of Thurston and Winkelnkemper [29], Giroux proved the following groundbreaking theorem regarding compatibilty of open books and contact structures:

Theorem 2.2 (Giroux [15]) Let $M$ be a closed oriented 3-manifold. Then there is a one-to-one correspondence between oriented contact structures on $M$ up to isotopy and open book decompositions of $M$ up to positive stabilizations and isotopy.

Considering contact 3-manifolds as boundaries of certain 4-manifolds together with some compatibility conditions is a current focus of research in low dimensional topology. From the contact topology point of view, it is the study of different types of fillings of a fixed contact manifold. In dimension four, there are essentially two considerations, yet we formulate them for all dimensions: Let $\left(X^{2 n}, \omega\right)$ be a symplectic manifold with cooriented nonempty boundary $M=\partial X$. If there exists a Liouville vector field (aka symplectic dilation) $v$ defined on a neighborhood of $\partial X$ pointing out along $\partial X$, then we obtain a positive contact structure $\xi$ on $\partial X$, which can be written as the kernel of contact 1 -form $\alpha=\left.\iota_{\nu} \omega\right|_{\partial X}$. When this holds, we say $(M, \xi)$ is the $\omega$-convex boundary or strongly convex boundary of $(X, \omega)$. For the sake of entirety, note when $v$ points inside, we obtain a negative contact structure instead, and in this case we say $(M, \xi)$ is the $\omega$-concave boundary of $(X, \omega)$.

Now if $\left(X^{2 n}, J\right)$ is almost-complex, then the complex tangencies on $M=\partial X$ give a uniquely defined oriented hyperplane field. It follows that there is a 1 -form $\alpha$ on $M$ such that $\xi=K e r \alpha$. We define the Levi form on $M$ as $\left.d \alpha\right|_{\xi}(\cdot, J \cdot)$. If this form is positive definite then $(M, \xi)$ is said to be strictly $J$-convex boundary of $(X, J)$, and if it is $J$-convex for an unspecified $J$ (for instance when $J$ is tamed by a given symplectic form), we say $(M, \xi)$ is strictly pseudoconvex boundary. If $(X, \omega, J)$ is an almost-Kähler manifold, i.e. a manifold equipped with a symplectic form $\omega$ and a 
compatible almost-complex structure $J$, then it can be shown that strict pseudoconvexity of the boundary is equivalent to the condition that $\left.\omega\right|_{\xi}>0$ in dimenson $2 n=4$. We would like to remark that all these definitions can be formulated in more generality for hypersurfaces in $X^{2 n}$, not necessarily for $\partial X$ only.

For detailed and comparative discussions of these concepts, as well as proofs of some facts mentioned in the next subsection, the reader can turn to Eliashberg-Gromov [10] and Etnyre [11]. Also for further basic notions from contact topology of 3-manifolds such as Legendrian knots, Thurston-Bennequin framing, or convex surfaces, which we will occasionally use in this paper, see for example Ozbagci-Stipsicz [25].

\subsection{Stein manifolds}

A smooth function $\psi: X \rightarrow \mathbb{R}$ on a complex manifold $X$ of real dimension $2 n$ is called strictly plurisubharmonic if $\psi$ is strictly subharmonic on every holomorphic curve in $X$. We call a complex manifold $X$ Stein, if it admits a proper strictly plurisubharmonic function $\psi: X \rightarrow[0, \infty)$ (after Grauert [19]). Thus a compact manifold $X$ with boundary which is equipped with a complex structure in its interior is called compact Stein if it admits a proper strictly plurisubharmonic function which is constant on the boundary.

Given a function $\psi: X \rightarrow \mathbb{R}$ on a Stein manifold, we can define a 2 -form $\omega_{\psi}=$ $-d J^{*} d \psi$. It turns out that $\psi$ is a strictly plurisubharmonic function if and only if the symmetric form $g_{\psi}(\cdot, \cdot)=\omega_{\psi}(\cdot, J \cdot)$ is positive definite. So every Stein manifold $X$ admits a Kähler structure $\omega_{\psi}$, for any strictly plurisubharmonic function $\psi: X \rightarrow$ $[0, \infty)$. It is easy to see that the restriction of $\omega_{\psi}$ to each level set $\psi^{-1}(t)$ gives a Levi form on $\psi^{-1}(t)$, implying that all nonsingular level sets of $\psi$ are strictly pseudoconvex hypersurfaces. Thus in this article, we equivalently call a Stein manifold a strictly pseudoconvex manifold. Moreover, it was observed in [10] that the gradient vector field of $\psi$ defines a (global) Liouville vector field $v=\nabla_{\psi}$, making all nonsingular level sets $\omega_{\psi}$-convex. Hence, Stein manifolds exhibit strongest filling properties for a contact manifold which can be realized as their boundary.

In this article, we are mainly interested in compact Stein surfaces. Another characterization of these manifolds, which might be called "the topologist's fundamental theorem of compact Stein surfaces", is due to Eliashberg, and was made explicit by Gompf in dimension four:

Theorem 2.3 (Eliashberg [8], Gompf [17]) A smooth oriented compact 4-manifold with boundary is a Stein surface, up to orientation preserving diffeomorphisms, if and only if it has a handle decomposition $X_{0} \cup h_{1} \cup \ldots \cup h_{m}$, where $X_{0}$ consists of 0 - and 
1-handles and each $h_{i}, 1 \leq i \leq m$, is a 2-handle attached to $X_{i}=X_{0} \cup h_{1} \cup \ldots \cup h_{i}$ along a Legendrian circle $L_{i}$ with framing $t b\left(L_{i}\right)-1$.

All structures we have introduced so far meet in the following theorem:

Theorem 2.4 (Loi-Piergallini [23], also see Akbulut-Ozbagci [2]) An oriented compact 4-manifold with boundary is a Stein surface, up to orientation preserving diffeomorphisms, if and only if it admits a PALF.

Throughout the article, we give ourselves the freedom of using the prefix 'anti' as a shorthand, whenever an oriented manifold $X$ admits a structure when the orientation on $X$ is reversed; like anti-symplectic, anti-Kähler, or anti-Stein. For Lefschetz fibrations and open books though, we use 'positive' and 'negative' adjectives to distinguish two possible cases.

\section{Simple folded symplectic structures}

The definition of symplectic (or anti-symplectic) structures can be enlarged as follows in order to cover a larger family of manifolds, which was shown by Cannas da Silva [5] to contain entire family of closed oriented smooth 4-manifolds:

Definition 3.1 A folded symplectic form on a smooth $2 n$-dimensional manifold $X$ is a closed 2-form $\omega$ such that $\omega^{n}$ is transverse to the 0 -section of $\Lambda^{2 n} T^{*} X$, and whenever this intersection is nonempty, $\omega^{n-1}$ does not vanish on the hypersurface $H=\left(\omega^{n}\right)^{-1}(0)$, called the fold.

For an oriented $X$, the kernel of $\omega$ on $H$ integrates to a foliation called null-foliation. Martinet's singular form $x_{1} d x_{1} \wedge d y_{1}+d x_{2} \wedge d y_{2}+\cdots+d x_{n} \wedge d y_{n}$ on $\mathbb{R}^{2 n}$ defines the standard folded symplectic structure, as every folded symplectic form can be expressed in this way in an appropriate Darboux coordinate system around any point on the fold. There is also a simple folded structure that every even dimensional sphere carries: We think of $S^{2 n}$ sitting in $\mathbb{R}^{2 n+1}$, then pull back the standard symplectic form $d x_{1} \wedge d y_{1}+\cdots+d x_{n} \wedge d y_{n}$ on the unit disk bounded by the equator in $\mathbb{R}^{2 n}$ to $S^{2 n}$ by the projection maps along the last coordinate, and finally glue them along the fold $S^{2 n-1}$ to obtain $\omega_{0}$. This is equivalent to doubling the unit disk equipped with its standard symplectic form (by reversing the orientation on one of the disks). We call this form the standard folded symplectic form on $S^{2 n}$.

For more on folded symplectic structures, the reader is referred to the work of Cannas da Silva, Guillemin and Woodward [5; 6]. Here we only consider these forms on 
Riemann surfaces and compact 4-manifolds, possibly with boundaries. For the former class, folded symplectic forms form an open and dense set in the space of 2-forms, whereas in dimension four openness remains but the nonvanishing condition implies that they are nongeneric. We say an embedded surface $\Sigma \subset X^{4}$ is a folded symplectic submanifold of $(X, \omega)$ if $\left.\omega\right|_{\Sigma}$ is a folded symplectic form on $\Sigma$. Observe that $S^{2}$ equipped with the standard form obtained by pulling back $d x_{1} \wedge d y_{1}$ embeds as a folded symplectic submanifold of $S^{4}$ with the standard folded symplectic form defined as the pullback of $d x_{1} \wedge d y_{1}+d x_{2} \wedge d y_{2}$ as above.

The following proposition provides several examples of folded symplectic 4-manifolds:

Proposition 3.2 Let $X$ be a closed oriented smooth 4 -manifold and $\Sigma$ be a closed oriented surface. If $f: X \rightarrow \Sigma^{2}$ is an achiral Lefschetz fibration such that the regular fiber is a closed oriented surface $F$ which is nonzero in $H_{2}(X ; \mathbb{R})$, then $X$ admits a folded symplectic structure $\omega$ such that fibers are symplectic and the fold $H$ is an $F$-bundle over $S^{1}$. The fold $H$ splits $X$ into pieces $X_{+}$and $X_{-}$, and $f$ induces symplectic Lefschetz fibrations on $\left(X_{+},\left.\omega\right|_{X_{+}}\right)$and on $\left(-X_{-},\left.\omega\right|_{X_{-}}\right)$, respectively. Furthermore, any finite set of sections can be made folded symplectic for an appropriate choice of $\omega$. This form is canonical up to deformation equivalence of folded symplectic forms.

We will call this type of folded symplectic structures simple (after Thurston [28]). Base spaces of the fibrations defined on $X_{+}$and $-X_{-}$are determined by an arbitrary splitting $\Sigma=\Sigma_{+} \cup \Sigma_{-}$. Here we take $\Sigma_{-}=D^{2}$ for simplicity. Observe that the fibration induces an exact sequence

$$
\pi_{1}(F) \rightarrow \pi_{1}(X) \rightarrow \pi_{1}(\Sigma) \rightarrow \pi_{0}(F) \rightarrow 0
$$

It follows that fibers are connected if the base is simply-connected. Otherwise we can define a new achiral Lefschetz fibration from $X$ to the finite cover of $\Sigma$ corresponding to the finite-index subgroup $f_{\#}\left(\pi_{1}(X)\right)$ in $\pi_{1}(\Sigma)$, which has connected fibers. Finally, one can perturb $f$ to get a fibration which has at most one critical point on each fiber. Hence, without loss of generality, we will assume that the fibers of $f$ are connected and critical values are distinct.

Proof of Proposition 3.2 Start by connecting all negative critical points in the base by an embedded arc in the complement of positive critical points, and cover it by the images of orientation reversing charts so that we get a closed neighborhood $\Sigma_{-} \cong D^{2}$ of this arc away from the positive critical points. This can be done because around the regular points we have freedom to take charts of either orientation. After we reverse the orientation on $f^{-1}\left(\Sigma_{-}\right)$, the map $f: f^{-1}\left(\Sigma_{-}\right) \rightarrow \Sigma_{-}$defines a negative Lefschetz 
fibration. Set $\Sigma_{+}=\Sigma \backslash \Sigma_{-}, C=\Sigma_{+} \cap \Sigma_{-}, X_{+}=f^{-1}\left(\Sigma_{+}\right), X_{-}=f^{-1}\left(\Sigma_{-}\right)$, and $H=f^{-1}(C)$. If there are no negative critical points, we can choose $\Sigma_{-}$as a small disk around a regular value which does not contain any critical values. Now let $\beta$ be a folded symplectic form on $\Sigma$ which folds over $C$, such that it is a positive area form on $\Sigma_{+}$and a negative area form on $\Sigma_{-}$. These forms always exist: For example take $S^{2}$ with its standard folded form $\omega_{0}$, and suppose $\Sigma_{ \pm}$has genus $g_{ \pm}$. Symplectic connect sum the upper-hemisphere of $S^{2}$ with a closed genus $g_{+}$surface equipped with a positive symplectic form, and the lower-hemisphere with a closed genus $g_{-}$ surface equipped with a negative symplectic form. This yields a folded symplectic form on $\Sigma$, folded along $C$.

We will construct a folded symplectic form on $X$ by mimicking Gompf's proof which generalizes Thurston's result for symplectic fibrations to symplectic Lefschetz fibrations (see Thurston [28] and Gompf-Stipsicz [18]). Let $\zeta$ be a closed 2-form on $X$ which evaluates positively on any closed surface contained in a fiber with the induced orientation. (We have not made any assumptions on the type of vanishing cycles, so one might have more than one closed surface on a fiber if there are separating vanishing cycles.) First we wish to define a closed 2-form $\eta$ on all over $X$ which is symplectic on each $F_{y}=f^{-1}(y)$, for all $y \in \Sigma$.

Let $A$ be a tubular neighborhood of $C$ in $\Sigma$ which does not contain any critical values. Choose disjoint open balls $U_{+, k}$ around each positive and $V_{-, l}$ around each negative critical point so that these sets do not intersect $f^{-1}(A)$ in $X$ and that in appropriate charts the fibration map can be written as $f\left(z_{1}, z_{2}\right)=z_{1} z_{2}$ and $f\left(z_{1}, z_{2}\right)=\bar{z}_{1} z_{2}$, respectively. Take the standard forms

$$
\omega_{+, k}=d x_{1} \wedge d y_{1}+d x_{2} \wedge d y_{2}=-\frac{i}{2} d z_{1} \wedge d \bar{z}_{1}-\frac{i}{2} d z_{2} \wedge d \bar{z}_{2}
$$

on $U_{+, k}$ and

$$
\omega_{-, l}=-d x_{1} \wedge d y_{1}+d x_{2} \wedge d y_{2}=\frac{i}{2} d z_{1} \wedge d \bar{z}_{1}-\frac{i}{2} d z_{2} \wedge d \bar{z}_{2}
$$

on $V_{-, l}$ for all $k, l$. For any $y \in f\left(U_{+, k}\right), F_{y} \cap U_{+, k}$ is a $J_{+, k}$-holomorphic curve, where $J_{+, k}$ is an almost-complex structure compatible with $\omega_{+, k}$. Similarly for any $y \in f\left(V_{-, l}\right), F_{y} \cap V_{-, l}$ is $J_{-, l}$-holomorphic curve, where $J_{-, l}$ is an almost-complex structure compatible with $\omega_{-, l}$. Having expressed $\omega_{+, k}$ and $\omega_{-, l}$ in terms of Kähler forms, we can take these almost-complex structures as $(i, i)$ and $(-i, i)$, respectively. It follows that $\left.\omega_{+, k}\right|_{F_{y} \cap U_{+, k}}$ is symplectic, so we can extend it to a symplectic form $\omega_{y}$ on the entire fiber and get $\omega_{y}$ defined for all points in each $f\left(U_{+, k}\right)$ this way. Do the same for all points in $f\left(V_{-, l}\right)$, for every $j$. Finally, for all remaining $y \in \Sigma$ take any symplectic form $\omega_{y}$ on the fiber, and rescale every $\omega_{y}$ we have defined away from all $U_{+, k}$ and $V_{-, l}$ so that they are in the same cohomology class as the restriction of $\zeta$ 
to each $F_{y}$. Next, cover $\Sigma$ with finitely many balls $B_{s}$ containing at most one critical value, and whenever they do contain a critical value, assume they are centered at that point. Reindex $U_{+, k}$ and $V_{-, l}$, and shrink them if necessary to make sure they lie in $f^{-1}\left(B_{S}\right)$ for some $s$. Define $\eta_{s}$ on each $f^{-1}\left(B_{S}\right)$ as the pullback of $\omega_{+, s}, \omega_{-, s}$, or $\omega_{y}$ by $r_{s}$, where $r_{s}$ is the retraction of $f^{-1}\left(B_{s}\right)$ to the fiber $F_{y}$ over the center of $B_{s}$, or the union of $F_{y}$ either with closure of $U_{+, s}$ or with closure of $V_{-, s}$, whenever $B_{S}$ contains a positive or negative critical value, respectively. Now we can glue these forms to construct the 2-form $\eta$ we wanted, by using a partition of unity and that each $\eta_{s}$ is cohomologous to $\left.\zeta\right|_{f^{-1}\left(B_{s}\right)}$ as in Gompf-Stipsicz [18].

We claim that $\omega_{\kappa}=\kappa \eta+f^{*}(\beta)$ is a folded symplectic form on $X$, where $\kappa$ is a small enough positive real number. $\omega_{\kappa}$ is clearly closed and symplectic in the fiber direction. It follows that for any noncritical point $x \in F_{y}, T_{x} X=T_{x} F_{y} \oplus\left(T_{x} F_{y}\right)^{\perp \eta}$. Here $f^{*}(\beta)$ is nondegenerate over $\left(T_{x} F_{y}\right)^{\perp \eta}$ for all $x \notin H$, implying that for sufficiently small $\kappa, \omega_{\kappa}$ is nondegenerate on $X \backslash\left(H \bigcup_{s}\left(U_{+, s} \cup V_{-, s}\right)\right)$. On the other hand $\left.\omega_{\kappa}\right|_{U_{+, s}}=\kappa \omega_{+, s}+f^{*}(\beta)$ and $\left.\omega_{\kappa}\right|_{V_{-, s}}=\kappa \omega_{-, s}+f^{*}(\beta)$. Therefore for any nonzero $v \in T U_{+, s}$, we have

$$
\omega\left(v, J_{+, s} v\right)=\kappa g(v, v)_{+, s}^{2}+\beta\left(f_{*}(v), i f_{*}(v)\right)>0,
$$

where $g(-,-)_{+, s}$ is the metric induced from $\omega_{+, s}$ and $J_{+, s}$. Likewise, for any nonzero $v \in T V_{-, s}$, we will have

$$
\omega\left(v, J_{-, s} v\right)=\kappa g(v, v)_{-, s}^{2}+\beta\left(f_{*}(v),-i f_{*}(v)\right)>0,
$$

$g(-,-)_{-, s}$ being the metric induced from $\omega_{-, s}$ and $J_{-, s}$. (Recall that $\beta$ is negative on $\Sigma_{-}$.) Hence $\omega_{\kappa}$ is symplectic everywhere on $X$ except $H$, where it vanishes transversely. Moreover, $f^{*}(\beta)$ is a folded symplectic form on any section, so taking $\kappa$ even smaller, we can as well assume that any finite collection of sections of $f$ are folded symplectic. It is easy to check that the folded symplectic form we get satisfies all the other declared properties. (Also see Remark 3.3).

The homological assumption in the theorem is a very mild one. If $S$ is the set of critical points of the achiral fibration $f: X \rightarrow \Sigma$, then the tangencies of the fibers define a complex line bundle $L=\operatorname{Ker}(d f)$ on $X \backslash S$, which extends uniquely over $X$. It follows that unless we have a torus fibration, the regular fiber $F$ is essential, since $\left\langle c_{1}(L), F\right\rangle=\chi(F)$. Also if the fibration is obtained from a pencil by blowing up the base points, the exceptional spheres will become sections of the fibration, guaranteeing that the fibers are essential in the homology.

Remark 3.3 Alternatively, the folded symplectic form in Proposition 3.2 can be constructed by using the folding operation described by Cannas da Silva, Guillemin 
and Woodward [6]. Restrictions of $\beta$ on $\Sigma_{+}$and on $\Sigma_{-}$give well-defined area forms $\beta_{+}$and $\beta_{-}$, respectively. Gompf's method can be used to define a symplectic form $\kappa_{+} \eta+f^{*}\left(\beta_{+}\right)$on $X_{+}$, where $\eta$ is a 2 -form on $X$ that restricts to the fibers as a (positive) symplectic form and $\kappa_{+}$is a small enough positive real number. The orientation on the base together with the orientation on the regular fiber determines the orientation of the total space, and thus by taking the opposite orientation on $\Sigma_{-}$ but keeping the orientation on $F$, one orients $-X_{-}$. Let $\bar{f}:-X_{-} \rightarrow \Sigma_{-}$be the fibration defined by taking orientation-preserving charts for $f: X_{-} \rightarrow \Sigma_{-}$, then we can define a symplectic form $\kappa_{-} \eta+\bar{f}^{*}\left(-\beta_{-}\right)$on $-X_{-}$(as $-\beta_{-}$is the area form on $\Sigma_{-}$) by following the same construction method. Observe that $\bar{f}^{*}\left(-\beta_{-}\right)=f^{*}\left(\beta_{-}\right)$. Hence, setting $\kappa=\min \left\{\kappa_{+}, \kappa_{-}\right\}$, we obtain two symplectic manifolds $\left(X_{+}, \omega_{+}\right)$and $\left(-X_{-}, \omega_{-}\right)$, where $\omega_{ \pm}=\kappa \eta+f^{*}\left(\beta_{ \pm}\right)$. Let $\iota_{ \pm}$be the inclusions of boundaries into $\pm X_{ \pm}$, then $\iota_{+}^{*}\left(\omega_{+}\right)=\kappa \eta=\iota_{-}^{*}\left(\omega_{-}\right)$and the orientations of both null-foliations agree. Thus we can glue these pieces to obtain a folded symplectic structure on $X_{+} \cup X_{-}=X$, which agrees with $\omega_{+}$and $\omega_{-}$in the complement of a tubular neighborhood of the fold $\partial X_{+}=H=-\partial X_{-}$. (See [6] for details.) This form is deformation equivalent to the form $\kappa \eta+f^{*}(\beta)$ in Proposition 3.2.

\section{Existence of folded symplectic structures on closed oriented 4-manifolds}

Here we show that any closed oriented smooth 4-manifold $X$ can be equipped with a folded symplectic form. For the sake of completeness, we start by outlining Etnyre and Fuller's proof [12] that every 4-manifold admits an achiral Lefschetz fibration after a surgery along a framed circle: Take a handlebody decomposition of $X$ with one 0 - and one 4-handle, let $X_{1}$ denote the union of the 0 -handle, 1-handles and 2handles, and $X_{2}$ denote the union of the 3-handles and the 4-handle. By Theorem 2.1 there exist achiral Lefschetz fibrations $f_{i}: X_{i} \rightarrow D^{2}$, which necessarily have bounded fibers, and stabilizing both fibrations we may as well assume the fibers have connected boundaries. After a possible slight modification of the handlebody decomposition, Etnyre and Fuller manipulate the contact structures on the boundaries so that they are both overtwisted and homotopic as plane fields. Then it follows from results of Eliashberg and Giroux that we have isotopic contact structures, and thus the induced open books are the same, possibly after some stabilizations and isotopies. Denoting the final manifolds and fibrations with $X_{i}$ and $f_{i}$ again, we may therefore assume that the open book decompositions induced by these fibrations on the common boundary $H=\partial X_{1}=-\partial X_{2}$ are the same, so we can glue both pieces of $X$ back along the 
truncated pages, and obtain an achiral Lefschetz fibration

$$
f_{1} \cup f_{2}: W=X_{1} \bigcup_{f_{1}^{-1}\left(\partial D^{2}\right)=f_{2}^{-1}\left(\partial D^{2}\right)} X_{2} \longrightarrow S^{2} .
$$

To recover $X$ we need to glue $S^{1} \times D_{1}^{2}$ to $S^{1} \times D_{2}^{2}$, where

$$
S^{1} \times D_{i}^{2}=\partial X_{i} \backslash f_{i}^{-1}\left(\partial D^{2}\right) .
$$

Filling the boundary of $W$ with an $S^{1} \times D^{3}$ gives the same result, so we can view $W$ as $X \backslash N$ where $N$ is a neighborhood of an embedded curve $\gamma \subset X$. Now, if we instead add on a $D^{2} \times S^{2}$ so that each $\partial D^{2} \times\{p t\}$ is identified with $S^{1} \times\{p t\}$, we can extend the fibration on $W$ by the projection on the $S^{2}$ component of $D^{2} \times S^{2}$. Hence, we obtain an achiral Lefschetz fibration over $S^{2}$ on the resulting manifold $Y$, where the section $S$ of this fibration discussed by Etnyre and Fuller [12] can be taken as $0 \times S^{2}$ coming from the glued in $D^{2} \times S^{2}$, implying $S$ has trivial normal bundle in $Y$.

We will refer the following as the standard model: Consider $S^{4}$ with the standard folded symplectic structure $\omega_{0}$ described before, and take $S^{4} \cap\left\{x_{4}=0\right\}$ vertical to the fold $H_{0}=S^{4} \cap\left\{x_{5}=0\right\}$. Take $S_{0}=S^{4} \cap\left\{x_{4}=0=x_{3}\right\} \cong S^{2}$ which intersects the fold along the circle $C_{0}=\left\{x_{1}^{2}+x_{2}^{2}=1 \mid x_{3}=x_{4}=x_{5}=0\right\}$. It is easy to see that $\omega_{0}$ restricts to this $S_{0}$ as the standard folded symplectic form on $S^{2}$, folded along $C_{0}$, and symplectic on the normal disks to $S_{0}$. Fix a disk neighborhood $M_{0}$ of $S_{0}$ so that $\omega_{0}$ evaluates as 1 on each normal disk. That is, each normal disk projects onto unit disk $\left\{x_{3}^{2}+x_{4}^{2} \leq 1 \mid x_{1}=x_{2}=x_{5}=0\right\}$ symplectomorphically. By restricting $\omega_{0}$, we get two folded symplectic manifolds $M_{0} \cong S^{2} \times D^{2}$ and $N_{0}=S^{4} \backslash M_{0} \cong D^{3} \times S^{1}$, with folds $S^{1} \times D^{2}$ and $D^{2} \times S^{1}$, respectively.

The existence of the section $s: S^{2} \rightarrow S \subset X$ guarantees that the fiber of the achiral Lefschetz fibration $f: Y \rightarrow S^{2}$ is homologically essential and therefore there exists a folded symplectic form $\omega$ as described in Proposition 3.2. This restricts to $Y \backslash M$, where $M \cong S^{2} \times D^{2}$ is a neighborhood of $S$. We may assume $\omega$ is constructed such that $M$ is identified with $M_{0}$ in the standard model above as follows: Let $\phi: M \rightarrow M_{0}$ be an orientation preserving diffeomorphism such that $\phi$ is orientation preserving on the spheres (and on the normal directions as well), and that it maps the upper-hemisphere of $S_{0}$ (where $\omega_{0}$ is positive) to the positive part of $S$. Then one can start the construction in the proof of Proposition 3.2 with the folded symplectic form $s^{*} \phi^{*}\left(\omega_{0}\right)$ on the base sphere, which naturally restricts to an area form on each hemisphere. We can also modify the symplectic form $\kappa \eta$ on the fibers so that it is symplectomorphic to $\phi^{*}\left(\omega_{0}\right)$ on the normal disks to $S$, each of which lies on a fiber. 
Hence we obtain a folded symplectic form $\omega$ on $X$ such that $\left(M,\left.\omega\right|_{M}\right)$ is folded symplectomorphic to $\left(M_{0},\left.\omega_{0}\right|_{M_{0}}\right)$. This allows us to trade $M$ for $N \cong S^{1} \times D^{3}$ and extend the folded symplectic structure to $(Y \backslash M) \cup N \cong X$. The effect of this surgery on the fold of $Y$ is to turn the surface fibration over $S^{1}$ into an open book decomposition on the resulting fold. The core curve of $N$ sits in the 3-manifold as the binding of this open book and therefore it carries a canonical framing. We have proved:

Theorem 4.1 Every closed oriented smooth 4-manifold $X$ admits a folded symplectic structure. Furthermore, there exist folded symplectic forms on $X$ with connected folds, such that a surgery along a framed curve which lies in the fold results in a simple folded symplectic manifold.

Remark 4.2 Away from the framed curve $\gamma$ in $X$, the folded symplectic model we have constructed is the restriction of the simple model discussed in the previous section, and as we will see shortly, the pieces are Stein and anti-Stein. So for any sort of pseudo-holomorphic curve counting with respect to this folded symplectic structure, the focus would be understanding the limit behaviors around $\gamma$ of the curves in the moduli space, where we do have a standard model, namely $\left(N_{0},\left.\omega_{0}\right|_{N_{0}}\right)$ above. (For a digression on this topic, see von Bergmann [30].) We would like to point out that both the knot type of $\gamma$ in the fold and its framing depend on the achiral Lefschetz fibrations we use in the construction, so does the simple model we get. The following example illustrates this phenomenon.

Example 4.3 If we construct $S^{4}$ following the recipe given in the proof of Theorem 4.1, we get $W=D^{2} \times D^{2} \bigcup_{C \times D^{2}} D^{2} \times D^{2}=S^{2} \times D^{2}$, which can be identified with $M_{0}$, and the simple folded symplectic form on $Y=S^{2} \times S^{2}=M_{0} \bigcup_{S^{2} \times \partial D^{2}} M_{0}$ can be constructed so that its restriction to each copy of $M_{0}$ is indeed the standard form $\omega_{0}$. Note that here both open books already agree, so we do not need to alter the contact structures and change the initial fibrations. Now if we undo the surgery, that is if we trade $N=N_{0}$ and $M$ in the proof, what we get is the standard folded symplectic form $\omega_{0}$ on $S^{4}$.

It is a standard fact that surgery along a framed curve in a simply-connected 4-manifold will result in connect summing with either $S^{2} \times S^{2}$ or $S^{2} \widetilde{\times} S^{2}$, depending on the framing, which can be thought as an element of $\pi_{1}(\mathrm{SO}(3))=\mathbb{Z}_{2}$. In Etnyre-Fuller [12] (also see Harer [20]) it is described how one can homotope the framed knot in the 3-manifold to another framed knot, which is isotopic in the ambient 4-manifold to the original one, so that their framings differ by one and that surgering the new curve yields an achiral Lefschetz fibration on the resulting manifold as well. Applying 

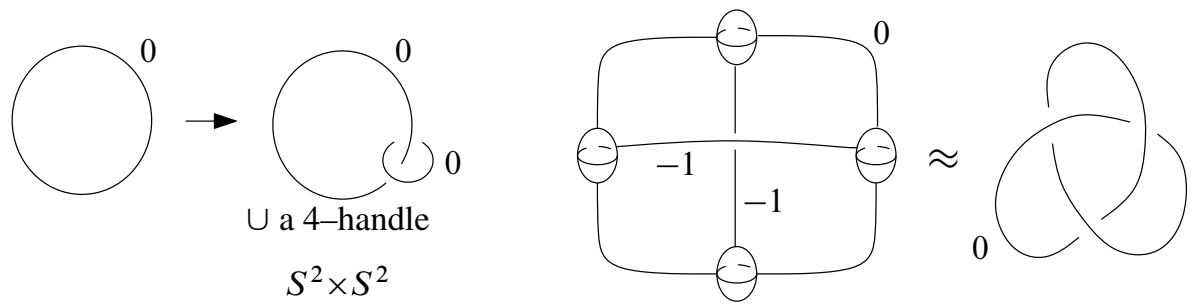

Figure 1: On the left: 0 -surgery along the binding yields a trivial $S^{2}$ fibration over $D^{2}$ on each piece, which make up $S^{2} \times S^{2}$. On the right: 0 -surgery along the new binding yields a cusp neighborhood on both sides.

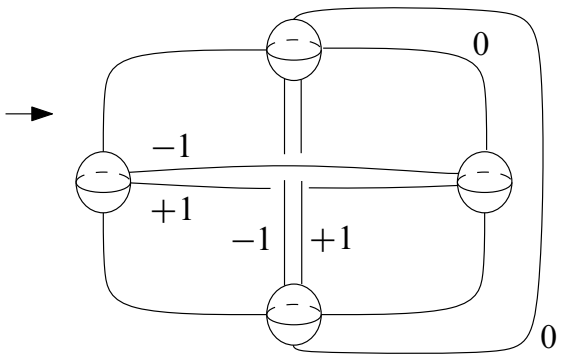

$\cup$ two 3-handles and a 4-handle

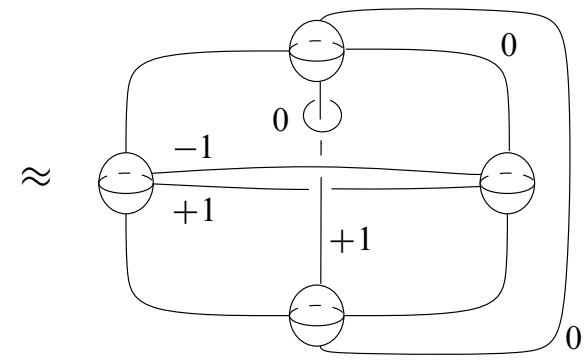

$\cup$ two 3-handles and a 4-handle
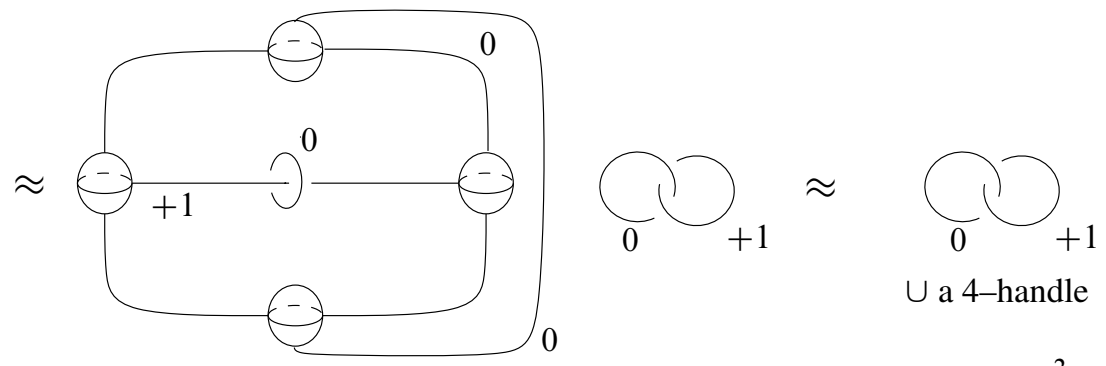

$\cup$ a 4-handle

$\cup$ two 3-handles and a 4-handle

Figure 2: The achiral Lefschetz fibration on the second associated model.

The total space is shown to be $\mathbb{C P} \mathbb{P}^{2} \overline{\mathbb{C P}}^{2}$.

this trick to our example, we can instead pass to an achiral Lefschetz fibration on $S^{2} \widetilde{\times} S^{2} \cong \mathbb{C P}^{2} \# \overline{\mathbb{C P}}^{2}$, which is a torus fibration with two cusp fibers of opposite signs (Figures 1 and 2). The monodromy of this achiral fibration is $t_{a} t_{b} t_{b}^{-1} t_{a}^{-1}$, and the corresponding Kirby diagram is depicted in Figure 2 (see Gompf [16]). To verify that 
this manifold is $\mathbb{C P} \mathbb{P}^{2} \# \overline{\mathbb{C P}}^{2}$, we first slide one of the vertical 2-handles over the other one, and then separate this pair from the rest of the diagram by sliding over the 0 framed 2-handle. Now the rest of the diagram can be shown to be $S^{4}$ after obvious handle cancelations. It is possible to see that our construction method will give a folded symplectic structure on $S^{4}$ equivalent to the standard one again. (Take the first example minus the neighborhood of the binding, do a pair of mirror stabilizations on each side, and then proceed as in the proof of Remark 6.3.) Note that the first simple model above is obtained by surgering the unknot, whereas the second comes from surgering the right trefoil in $S^{3}$. Surgery framings on them differ by one in $S^{4}$.

\section{Decomposition theorem}

While we shift our attention to Stein structures, we would like to have only nonseparating vanishing cycles in our constructions, as it is suggested by the correspondence between PALFs and compact Stein surfaces established by Loi and Piergallini [23] and by Akbulut and Ozbagci [2]. We start with the following lemma:

Lemma 5.1 Let $X$ be a closed oriented 4-manifold. Then it can be decomposed into two handlebodies, each of which admits an allowable achiral Lefschetz fibration over $D^{2}$, such that the fibers have connected boundaries and that the induced open books are the same.

Proof We follow the construction of Etnyre and Fuller with more care given to having fibrations allowable. Take a handlebody decomposition of $X$ with one $0-$ and one 4-handle, let $X_{1}$ be the union of the 0 -handle, 1-handles and 2-handles, and $X_{2}$ be the union of 3-handles and the 4-handle. As it was implicitly present in the thesis of Harer [20], and was also observed by Akbulut and Ozbagci [2], one can always build an achiral Lefschetz fibration on a given 2-handlebody so that all vanishing cycles are non-separating. Therefore, we can start with allowable fibrations and then proceed with stabilizations as described by Etnyre and Fuller [12] to match the induced open books. A stabilization is given by gluing a positive or a negative Lefschetz 2-handle along a new 1-handle added to a regular fiber, and in order to keep the binding connected, we always introduce another adjacent stabilization. Therefore, all vanishing cycles introduced during stabilizations are also nonseparating. The proof is completed by induction.

Theorem 5.2 Let $X$ be a closed oriented smooth 4-manifold. Then $X$ admits a decomposition into two codimension zero submanifolds $X_{+}$and $X_{-}$, such that $X_{+}$ 
and $-X_{-}$are both compact Stein manifolds with strictly pseudoconvex boundaries. These Stein structures can be chosen so that the induced contact structures $\xi_{+}$on $\partial X_{+}$ and $\xi_{-}$on $-\partial X_{-}$are isotopic. Furthermore, there are PALFs on each piece such that the open book decompositions they induce on $\partial X_{+}$and $-\partial X_{-}$are compatible with $\xi_{+}$ and $\xi_{-}$, respectively, and they coincide. In short, all data match on the hypersurface $H=\partial X_{+}=-\partial X_{-}$.

Proof The lemma above gives us a decomposition of $X$ into two pieces $X_{1}$ and $X_{2}$ with allowable achiral Lefschetz fibrations $f_{1}$ and $f_{2}$ on them, such that induced open books on the boundaries match. As in the proof of Theorem 4.1, we glue these two pieces along the truncated pages to get

$$
W=X_{1} \bigcup_{f_{1}^{-1}\left(\partial D^{2}\right)=f_{2}^{-1}\left(\partial D^{2}\right)} X_{2} .
$$

Next we glue in $S^{2} \times D^{2}$ to cap off the fibers and establish an achiral Lefschetz fibration $\hat{f}: Y \rightarrow S^{2}$ with closed fibers.

We wish to split the base of this fibration into two disks $D_{+}$and $D_{-}$so that all the positive critical values lie in the interior of $D_{+}$and all the negative ones lie in the interior of $D_{-}$. As discussed earlier, restrictions of $f$ give a positive Lefschetz fibration on $X_{+}=f^{-1}\left(D_{+}\right)$and a negative Lefschetz fibration on $X_{-}=f^{-1}\left(D_{-}\right)$, respectively. It also prescribes a surface bundle over $S^{1}=\partial D_{+}=-\partial D_{-}$on the hypersurface separating $Y_{+}$and $Y_{-}$. This time we would like to describe the splitting more carefully by taking into consideration how the global monodromies of the new fibrations are related to the original ones.

Let $\mu_{1}$ be the monodromy of the achiral Lefschetz fibration $f_{1}$ on $X_{1}$ and $\mu_{2}$ be the monodromy of the fibration $f_{2}$ on $X_{2}$. Fix a representation of $\mu_{1}$ by using arcs $s_{1}^{1}, \ldots, s_{k_{1}}^{1}$ and a representation of $\mu_{2}$ by using arcs $s_{1}^{2}, \ldots, s_{k_{2}}^{2}$. Corresponding critical values are denoted by $y_{i}^{1}$ and $y_{j}^{2}$. Monodromies of the open book decompositions bounding each fibration are given by $\mu_{1}$ and $\mu_{2}$ as well, and they coincide under an orientation reversing diffeomorphism, so $\mu_{1}=\left(\mu_{2}\right)^{-1}$. Let $V$ be a small neighborhood of a regular value in the base $S^{2}$. We obtain an achiral Lefschetz fibration

$$
f: W \backslash f^{-1}(V) \longrightarrow D=S^{2} \backslash V,
$$

which closes up to a fibration over $S^{2}$. If $g$ is the genus of the page, then this fibration is determined by the relation $\mu_{1} \mu_{2}=1$ in $\Gamma_{g, 1}$ and is mapped under the maps $\Gamma_{g, 1} \rightarrow \Gamma_{g}^{1} \rightarrow \Gamma_{g}$ to the relation that describes the achiral Lefschetz fibration

$$
\widehat{f}: Y \backslash \hat{f}^{-1}(V) \rightarrow D .
$$

Algebraic ${ }^{6} \mathcal{G}$ Geometric Topology, Volume 6 (2006) 

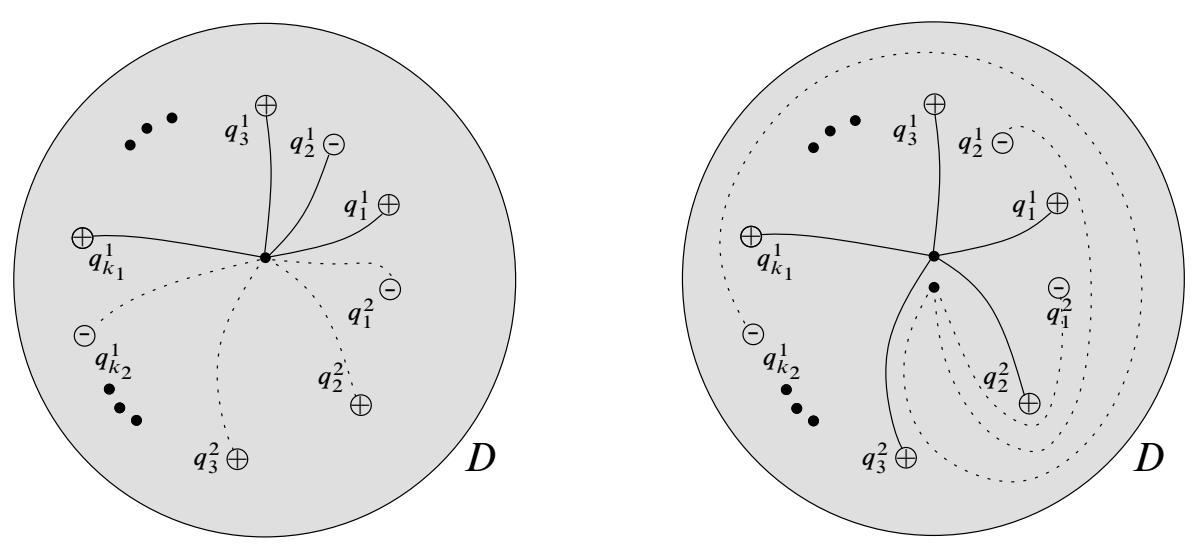

Figure 3: New monodromies from old ones. On the left: $\mu_{1}$ is given by solid arcs, and $\mu_{2}$ by dotted ones. On the right: Solid arcs are the positive arcs representing $\mu_{+}$, whereas conjugated dotted arcs are negative, providing a representation of $\mu_{-}$after closing the base back to $S^{2}$.

Since this map factors through $\Gamma_{g}^{1}$, the achiral Lefschetz fibration $\hat{f}$ comes naturally with a section $S$ of self-intersection zero. We denote images of the elements in $\Gamma_{g, 1}$ under this map with the same elements, so $\mu_{1} \mu_{2}=1$ is the global monodromy of the achiral Lefschetz fibration on $Y \backslash \hat{f}^{-1}(V)$. Note that we can use the same arcs $s_{i}^{1}$ and $s_{j}^{2}$ to represent the global monodromy of this fibration. Now, if we move counterclockwise and choose only the arcs that run through positive Dehn twists, we establish a monodromy $\mu_{+}$. Call these arcs positive. Next, we choose a nearby base point, and move counterclockwise by running through the negative Dehn twists only, while avoiding intersecting any positive arc. This way, we obtain a monodromy $\mu_{-}$. The new set of arcs involved in this monodromy will be referred as negative arcs. Observe that each negative arc is obtained by traveling around some old $\operatorname{arcs} s_{i}^{1}$ and $s_{j}^{2}$ in order to avoid intersecting positive arcs, then going around the aimed negative critical point once, and finally going all the way back on the same detour (Figure 3 ). That is, each negative arc corresponds to a conjugate of a negative Dehn twist in $\Gamma_{g}$, which defines a negative Dehn twist, too. By taking regular neighborhoods of these arcs such that positive and negative arcs stay apart, we get a disk enclosing only positive critical points, and an annulus containing only negative critical points. Closing the fibration to a fibration over $S^{2}$, the latter becomes a disk as well. Now we can enlarge any one of these disks so both disks share the same boundary, and call the one containing positive values $D_{+}$, and the other one $D_{-}$. So we have a new factorization of the global monodromy of $\hat{f}$, given by the relation $\mu_{+} \mu_{-}=1$. The section $S$ prescribes how to lift the new elements $\mu_{ \pm}$of $\Gamma_{g}$ to $\Gamma_{g}^{1}$ uniquely. 
We proceed with taking out the tubular neighborhood $S^{2} \times D^{2}$ of the section from $Y=Y_{+} \cup Y_{-}$, and we get an inherited splitting $X_{+} \cup X_{-}$. The discussion above shows that $\mu_{+}$defines a positive Lefschetz fibration on $X_{+}$and $\mu_{-}$defines a negative fibration on $X_{-}$. To recover the original 4-manifold $X$ we need to put back in $S^{1} \times D^{3}$, which has the same effect as gluing each other the tubular neighborhoods $S^{1} \times D_{+}^{2}$ and $S^{1} \times D_{-}^{2}$ of the bindings of open books on $\partial X_{+}$and $\partial X_{-}$, respectively. Therefore we can think of $X$ as decomposing into $X_{+}$and $X_{-}$. We claim that this decomposition possesses the desired properties.

When we take out a tubular neighborhood of $S$ from $Y$, we turn the positive and negative Lefschetz fibrations on $Y_{+}$and $Y_{-}$into a PALF on $X_{+}$and a NALF on $X_{-}$, respectively. In the meantime the surgery converts the surface fibration that separates $Y_{+}$ and $Y_{-}$to an open book decomposition on the common boundary $H=\partial X_{+}=-\partial X_{-}$. The binding of this open book is the identified bindings of $\partial X_{+}$and $-\partial X_{-}$, the page $F$ is the bounded closed surface obtained by cutting off a disk from the regular fiber of $\hat{f}$, and the monodromy is induced from the fibration on either side. Noting that the NALF on $X_{-}$becomes a PALF on $-X_{-}$, we see that both PALFs induce the same open book decomposition on their boundaries.

By Theorem 2.4, both $X_{+}$and $-X_{-}$admit Stein structures. We will construct these Stein structures using Eliashberg's characterization so that they match on the common boundary. The technique we are going to use is the same as the one which was presented by Akbulut and Ozbagci [2]: The PALF on $X_{+}$is obtained by attaching positive Lefschetz handles $h_{1}, \ldots, h_{m}$ to $X_{0}=F \times D^{2}$, which has the obvious PALF defined by projection onto $D^{2}$ component. The same is true for the PALF on $-X_{-}$. $F \times D^{2}$ has a natural Stein structure by Theorem 2.3. We can assume all vanishing cycles (coming from either side) sit in various pages of the open book on $H$. Read backwards, we can think of the fibrations as being constructed by attaching positive and negative Lefschetz handles to $H$ on either side in a sequence following the monodromy of the open book. Thus we can induct on the number of handles. Assume that the PALF on $X_{i-1}=X_{0} \cup h_{1} \cup \ldots \cup h_{i-1} \quad(i \leq m)$ induces an open book decomposition on its boundary, and it carries a Stein structure such that the contact structure induced on the boundary is compatible with this open book. Let $C$ be the vanishing cycle of the positive Lefschetz handle $h_{i}$ lying on a page $F$ of $\partial X_{i-1}$. We open up the open book decomposition and choose a page against $F$, and glue them together along the binding $B$ to form a smooth closed convex surface $\Sigma$ in the 3 -manifold $\partial X_{i-1}$. As $C$ is nonseparating, $\Sigma \backslash C$ is connected and it contains the dividing set, namely $B$. So we can use the Legendrian realization principle (see Giroux [14] and Honda [21]) to isotope $\Sigma$ through convex surfaces to make $C$ Legendrian. Note that this is done by a small $C^{\infty}$ isotopy of the contact structure supported in a neighborhood of $\Sigma$, which fixes the 
binding pointwise. Hence the framing of $C$ relative to the fiber $F$ is the same as its contact framing, implying that the Lefschetz handle $h_{i}$ is attached along a Legendrian curve with framing $t b-1$. By Theorem 2.3 the Stein structure extends over this handle, and as shown by Gay in [13], the new open book on $\partial X_{i}$ will be compatible with the new induced contact structure on $\partial X_{i}$. This completes the induction. Repeating the same argument dually for $-X_{-}$, we see that the compatible open books on $\partial X_{+}$and $\partial\left(-X_{-}\right)$are isotopic, and therefore the induced contact structures $\xi_{+}$on $\partial X_{+}$and $\xi_{-}$ on $\partial\left(-X_{-}\right)=-\partial X_{-}$are isotopic as well. So we fulfill all the matching conditions listed in the statement of the theorem.

Remark 5.3 Akbulut and Matveyev [1] asked if one could decompose a given closed oriented smooth 4-manifold into Stein pieces so that the induced contact structures on the separating 3-manifold coincide. Our theorem gives an affirmative answer to this question. In the same article authors remark that it is possible to alter their Stein decomposition to make the induced contact plane distributions homotopic, but the tightness of the contact structure precludes the use of Eliashberg's celebrated theorem on overtwisted contact structures to conclude more. Considering the underlying PALFs and isotopies of open books gives a way around this difficulty, thanks to Giroux's Theorem.

Remark 5.4 In [27], Quinn studied so-called dual decompositions of 4-manifolds: descriptions of 4-manifolds as a union of two 2-handlebodies. The author formulates the same question as in [1] in terms of necessary sequence of Kirby moves to relate a possibly nonmatching Stein decomposition. Theorem 5.2 provides an implicit answer to this question, and we would like to take this as an opportunity to summarize the handle calculus behind our construction: An arbitrary Stein decomposition $X=X_{1} \cup X_{2}$ comes with some PALF pair. Using the stabilization moves of Etnyre and Fuller, we first change this PALF pair with a matching pair. This corresponds to adding canceling 1 - and 2-handles to each $X_{i}$, or in other words, we add canceling handle pairs of index 1,2 and 3 in the original handlebody decomposition of $X$. In the next step, we pass to a cobordant 4-manifold $Y$ so that we can split the positive and negative Lefschetz handles. Then we 'undo' the surgery and get the decomposition $X=X_{+} \cup X_{-}$with Stein structures on each piece that coincide on the common boundary. Having the simply-connected case in mind, this intermediate step can be seen as a stabilization. Let $W \cong X \backslash S^{1} \times D^{3}$ be the complement of a regular neighborhood of the framed knot $\gamma$ in $X$, then the first surgery defines a cobordism

$$
[0,2] \times W \bigcup_{[0,2] \times S^{1} \times S^{2}}\left([0,1] \times S^{1} \times D^{3}\right) \cup_{1 \times S^{1} \times S^{2}}\left([1,2] \times D^{2} \times S^{2}\right),
$$

Algebraic 83 Geometric Topology, Volume 6 (2006) 
which is identity on the first component. We trade 2-handles of $X_{1}$ and $X_{2}$ in $Y$ by making use of the two extra handles of index 2. Finally, the composition of two cobordisms that gives back $X$ can be seen as the double of the cobordism above, and thus it deformation retracts to

$$
W \bigcup_{S^{1} \times S^{2}}\left(S^{4} \cup_{D^{2} \times S^{2}} S^{4}\right)
$$

This cobordism is built by attaching cells to $\partial W=S^{1} \times S^{2}$, where $D^{2} \times S^{2}$ is attached uniquely and the framing of $\gamma$ indicates in which one of the two ways we shall glue the other two $S^{1} \times D^{3}$ pieces. Although here we started with a (nonmatching) Stein decomposition, it is clear that the same discussion can be carried out in our main construction as well. Therefore we have a well-defined process, during which we first inflate the number of handles in a given decomposition of $X$, and then trade some of the 2-handles through a cobordism to achieve the desired decomposition at the end.

\section{Folded Kähler structures}

Unlike symplectic structures, random folded symplectic structures do not need to bear any information about the geometry or topology of the manifold they are defined on. In order to specify more meaningful members of this family, one first of all needs to impose some boundary conditions on the folding hypersurface. We would like to acknowledge a result of Kronheimer and Mrowka: In [22], the authors prove that a compact symplectic 4-manifold $(Y, \omega)$ with strictly pseudoconvex boundary has $S W_{Y}(K)=1$, where $K$ is the canonical class of $\omega$. This motivates us to see such manifolds as building blocks of 4-manifolds, and yields a good boundary constraint for folded symplectic structures, at least in this dimension. Henceforth, we assume that the fold $H=\left(\omega^{n}\right)^{-1}(0)$ of a given folded symplectic manifold $\left(X^{2 n}, \omega\right)$ is always connected and nonempty. We will generalize the notion of a Kähler structure on a smooth $2 n$-manifold by considering a distinguished subset of the family of folded symplectic structures, and we then present some properties of these structures:

Definition 6.1 A folded symplectic form $\omega$ on an oriented $2 n$-dimensional manifold $X$ is called a folded Kähler structure, if there is a tubular neighborhood $N$ of $H$ such that:

(1) The closure of each component of $X \backslash N$ is a compact Kähler manifold

$$
\left( \pm X_{ \pm},\left.\omega\right|_{ \pm X_{ \pm}}\right)
$$

with strictly pseudoconvex boundary, 
(2) $\left(N,\left.\omega\right|_{N}\right)$ is folded symplectomorphic to $\left([-1,1] \times H, d\left(\left(t^{2}+1\right) \pi^{*}(\alpha)\right)\right.$, where $\alpha$ is a contact 1 -form on the fold $H$, and $\pi$ is the projection $\pi:[-1,1] \times H \rightarrow H$.

In addition, if each $\left( \pm X_{ \pm},\left.\omega\right|_{ \pm X_{ \pm}}\right)$is strictly pseudoconvex, we say $\omega$ is a nicely folded Kähler structure on $X$.

In the definition above, nice folding can be reformulated as folding Stein manifolds along matching strictly pseudoconvex boundaries. Recall that if $\psi$ is a proper strictly plurisubharmonic function on a complex manifold $S$, then the associated 2-form $\omega_{\psi}=$ $-d J^{*} d \psi$ is Kähler, and importantly, the symplectic class of $\left(X, \omega_{\psi}\right)$ is independent of the choice of $\psi$ (see Eliashberg and Gromov [10]). Therefore, to complete our alternative formulation, we ask that each piece $\pm X_{ \pm}$should admit some proper strictly plurisubharmonic function $\psi_{ \pm}$, so that $\left.\omega\right|_{ \pm X_{ \pm}}=\omega_{\psi_{ \pm}}$. In short, it is built in the definition that a nicely folded Kähler manifold is folded Kähler. Finally note that, due to a theorem of Bogomolov [4], any compact folded Kähler manifold $X$ can be made nicely folded after deforming the complex structure and blowing down any exceptional curves. Even though these definitions narrow the family of folded symplectic structures quite a lot, it is important to note, at least in dimension four, that we still have an adequately large family in the light of the following result:

Theorem 6.2 Any closed oriented 4-manifold $X$ admits a nicely folded Kähler structure.

Proof By Theorem 5.2, $X$ can be decomposed into two compact Stein manifolds $X_{+}$ and $-X_{-}$with strictly pseudoconvex boundaries such that both induce the same contact structure on the common boundary $H=\partial X_{+}=-\partial X_{-}$. We begin with adding collars $\pm U_{ \pm}$to $\left( \pm X_{ \pm}, \omega_{ \pm}\right)$, and extending the symplectic structures to $\omega_{ \pm}^{\prime}$ on $\pm X_{ \pm}^{\prime}=$ $\pm\left(X_{ \pm} \cup U_{ \pm}\right)$so that new boundaries $\partial\left( \pm X_{ \pm}^{\prime}\right)$ are still convex and contactomorphic. Let $\xi_{ \pm}$be the induced contact structures on $\partial\left( \pm X_{ \pm}^{\prime}\right)$ and $\psi$ be a contactomorphism between them. Using the symplectic cut-and-paste argument of Etnyre [11], we can add a symplectic collar to $\left(\partial X_{+}^{\prime}, \omega_{+}^{\prime}\right)$ so that the new boundary is not only contactomorphic to $-\partial X_{-}^{\prime}$ but also the induced contact forms agree up to a multiple $k \in \mathbb{R}^{+}$. For the sake of brevity, let us assume that $U_{+}$above contains this collar part as well. So after rescaling $\omega_{-}^{\prime}$ (and $\omega_{-}$) by $k$ if necessary, we see that restrictions of symplectic forms $\left.\omega_{+}^{\prime}\right|_{\partial X_{+}^{\prime}}$ and $\left.k \omega_{-}^{\prime}\right|_{-\partial X_{-}^{\prime}}$ agree via $\psi$, and orientations of null-foliations (which correspond to Reeb directions) are the same. Therefore, once again we can apply the folding technique of Cannas da Silva, Guillemin and Woodward [6] to obtain a folded symplectic structure $\omega$ on $X_{+}^{\prime} \cup X_{-}^{\prime}$ such that $\omega$ agrees with $\omega_{ \pm}^{\prime}$ on the complement of a small tubular neighborhood of the fold $H$. We enlarge this neighborhood to include 
$U_{+}$and $U_{-}$and call it $N$. It follows that $X=X_{+} \cup N \cup X_{-} \cong X_{+} \cup X_{-}$, and $\left.\omega\right|_{X_{+}}=\omega_{+}$, whereas $\left.\omega\right|_{X_{-}}=k \omega_{-}$. Also note that, the folding operation provides us with the desired local model on $N$, that is, $\left(N,\left.\omega\right|_{N}\right)$ is folded symplectomorphic to $\left([-1,+1] \times H, d\left(\left(t^{2}+1\right) \pi^{*}(\alpha)\right)\right.$ by construction [6].

Lastly, suppose $\psi_{ \pm}: \pm X_{ \pm} \rightarrow[0, \infty)$ are proper strictly plurisbuharmonic functions such that $\pm \partial X_{ \pm}$correspond to the maximum points of $\psi_{ \pm}$, and $\omega_{ \pm}=-d J^{*} d \psi_{ \pm}$, respectively. If $k \neq 1$, we can replace $\psi_{-}$with $k \psi_{-}$and obtain $k \omega_{-}$above as a Kähler form of a strictly pseudoconvex manifold. Equipped with these properties, $\omega$ is a nicely folded Kähler form on $X$.

Remark 6.3 It is clear that Theorem 6.2 is a refinement of Theorem 4.1. Since the folded forms we have constructed in both proofs are obtained through similiar steps, one expects that these structures are actually equivalent. Next, we verify this fact, and this way we get an insight of how folded Kähler forms are 'supported' (precise definition is given below) by Lefschetz fibrations as was illustrated in Proposition 3.2:

Take the PALF on $X_{+}$in the proof of Theorem 4.1, and attach a symplectic 2-handle along the binding of the induced open book on $\partial X_{+}$as described by Eliashberg in [9]. This yields a symplectic Lefschetz fibration over $D_{+}^{2}$. Dually the same argument for the NALF on $X_{-}$gives an anti-symplectic Lefschetz fibration over $D_{-}^{2}$, and these handle attachments can be done so that two fibrations agree on the common boundary. Moreover, we can assume that these fibrations have genus at least two, so the fibrations can be matched as symplectic surface fibrations over a circle, as it was pointed out in [9]. At the end we get a simple folded symplectic manifold $Y$ obtained from $X$ after a surgery along a framed curve $\gamma$. However, any two simple folded symplectic forms compatible with a fixed achiral Lefschetz fibration are deformation equivalent by Proposition 3.2. Moreover, we can normalize both forms on the disks which are parallel copies of cocores of new 2-handles that were used to cap off the fibers. Hence, these two folded forms are deformation equivalent on $Y \backslash S^{2} \times D^{2}$. As the folded symplectic structure on $D^{3} \times S^{1}$ which is glued back in to recover $X$ is standard, the folded symplectic form constructed in the proof of Theorem 4.1 and the folded Kähler form obtained in Theorem 6.2 are indeed equivalent as folded symplectic structures.

Motivated by symplectic and near-symplectic cases (see Donaldson [7] and AurouxDonaldson-Katzarkov [3]), we can conclude our discussion above by defining the Lefschetz fibration analogue for our structures:

Definition 6.4 Let $X$ be a closed oriented 4-manifold, and decompose $S^{2}$ as the union of the upper-hemisphere $D_{+}$and the lower-hemisphere $D_{-}$which are glued 
along the equator $C=\partial D_{+}=-\partial D_{-}$. Then a smooth map $f: X \rightarrow S^{2}$ is said to be a folded Lefschetz fibration on $X$, if it restricts to a PALF over $D_{+}$, to a NALF over $D_{-}$, and to an open book over $C$ bounding both fibrations.

Definition 6.5 Let $X$ be a closed oriented 4-manifold equipped with a nicely folded Kähler form $\omega$. Then a folded Lefschetz fibration $f: X \rightarrow S^{2}$ is said to be compatible with $\omega$ if each Stein piece $X_{ \pm}$corresponds to $f^{-1}\left(D_{ \pm}\right)$, and if the contact structure they induce on $H=f^{-1}(C)$ is compatible with the open book decomposition coming from the fibration $f$. In this case, we also say that nicely folded Kähler manifold $(X, \omega)$ is supported by the folded Lefschetz fibration $f$.

The compatibility in the above definition is completely on the symplectic level. This becomes more visible if once again we recall that surgering the binding $\gamma$ of the open book $\left.f\right|_{H \backslash \gamma}: H \backslash \gamma \rightarrow S^{1}$, we pass to a simple model where the folded Lefschetz fibration can be extended to a folded symplectic achiral Lefschetz fibration $\hat{f}$ with closed fibers. Also note that, since Stein manifolds harbor less topological obstructions in complex dimensions $>2$, it is very likely that they admit higher dimensional analogues of PALFs with similar topological correspondences. If that is established, last two definitions, as well as several results in this paper, can be generalized to all $2 n$-dimensions.

The complete statement of Theorem 5.2 combined with Theorem 6.2 shows that, given a closed oriented 4-manifold $X$, one can always find a nicely folded Kähler structure $\omega$ on $X$ together with a compatible folded Lefschetz fibration. Next, we prove that this property in fact holds for any nicely folded Kähler structure:

Proposition 6.6 Any nicely folded Kähler structure $\omega$ on $X$, up to orientation preserving diffeomorphism, admits a compatible folded Lefschetz fibration.

Proof Each Stein piece $X_{+}$and $-X_{-}$admits a PALF by Theorem 2.4. If we construct these fibrations following the algorithm of Akbulut and Ozbagci [2] and keep track of the associated open books, the work of Plamenevskaya [26] shows that we can establish PALFs $f_{ \pm}: \pm X_{ \pm} \rightarrow D^{2}$ with the property that the open book decomposition on the boundaries are compatible with the contact structures induced from the Stein structures on $\pm X_{ \pm}$, respectively. As the contact structures are assumed to be the same, Theorem 2.2 tells us that we can match these open books after positive stabilizations. Consequently, we get a compatible folded Lefschetz fibration.

Remark 6.7 A folded Lefschetz fibration that supports a given folded Kähler structure fails to be unique. In fact, one can find infinitely many pairwise inequivalent such 
fibrations. This can be seen for example from the construction of [2], by using different $(p, q)$ torus knots in their algorithm which we adopt for building our achiral Lefschetz fibrations.

Example 6.8 The easiest examples are doubles. If $Y^{4}$ is a compact Kähler manifold with strictly pseudoconvex boundary, then $X=Y \cup-Y$ is equipped with a folded Kähler structure. When $Y$ is indeed Stein, we get a nicely folded structure. The first folded structure constructed in Example 4.3 is the double of standard $D^{4} \subset \mathbb{C}^{2}$, whereas the latter is a 'monodromy double' of a cusp neighborhood minus a section. Here by 'monodromy double' we mean that the pieces are first glued along the pages of the open books, and if the monodromy of the folded Lefschetz fibration on one piece is $\mu$, then the monodromy on the other one is $\mu^{-1}$.

Example 6.9 There is a construction which also allows us to see the nicely folded Kähler structure together with a compatible folded Lefschetz fibration. Take a contact $3-$ manifold $(H, \xi)$, and fix a positive open book decomposition $(B, f)$ compatible with $\xi$. Different PALFs bounding this open book describe (possibly) different Stein fillings of $(H, \xi)$. Indeed there are examples of infinitely many pairwise non-diffeomorphic contact 3-manifolds each of which admit infinitely many pairwise non-diffeomorphic Stein fillings constructed this way (see Ozbagci and Stipsicz [24]). Thus for every pair of PALFs $X_{1}$ and $X_{2}$ that fill the same open book, we can construct a nicely folded Kähler form on $X=X_{1} \cup-X_{2}$, as designated in the proof of Theorem 6.2.

Example 6.10 The main steps of our construction are depicted in the following simple, albeit instructive example: Start with classical handlebody decomposition of $X=\#_{8} S^{2} \times S^{2}$ with one 0 -handle, sixteen 2-handles, and a 4-handle. Let $X_{1}$ be the union of $0-, 2-$ handles, and $X_{2}$ be the 4 -handle. Each piece admits a $D^{2}$ fibration over $D^{2}$. However we wish to construct allowable fibrations, so we introduce two canceling 1- and 2-handle pairs and two canceling 2- and 3-handle pairs in the original handlebody decomposition of $X$. We start building the fibrations from the scratch: Add the 1-handles to the 0 -handle and 3-handles to the 4-handle. Attach the two canceling 2-handles with framing -1 to the union of the 0 - handle and 1 - handles. Attach the other two the same way to the handlebody $X_{2}$, which is the union of 3 handles and the 4 - handle. To simplify our description, we will label the 1-handles of the first handlebody as $a$ and $b$, which generate $\pi_{1}$ of the torus fiber with one boundary component, and we do the same for the 1-handles of $X_{2}$ under the obvious identification. So we obtain two achiral Lefschetz fibrations over disks with bounded torus fibers; one with monodromy $t_{a}^{-1} t_{b}^{-1}$, and one with $t_{b} t_{a}$. One can verify by Kirby calculus that each time we insert a pair of Lefschetz handles prescribed by $t_{a} t_{a}^{-1}$ or 
$t_{b} t_{b}^{-1}$, we introduce an $S^{2} \times S^{2}$. (See Figure 2, and observe that here we slide off the 2 -handle pair over a +1 framed 2-handle instead.) Doing this consecutively, we attach all the remaining 2-handles to the first handlebody, and obtain an achiral Lefschetz fibration on $X_{1}$ with monodromy

$$
\mu_{1}=t_{a}^{-1} t_{b}^{-1} t_{b} t_{a} t_{b} t_{a} t_{b} t_{a} t_{b} t_{a} t_{a}^{-1} t_{b}^{-1} t_{a}^{-1} t_{b}^{-1} t_{a}^{-1} t_{b}^{-1} t_{a}^{-1} t_{b}^{-1},
$$

whereas $X_{2}$ still has the monodromy

$$
\mu_{2}=t_{b} t_{a}=\left(t_{a}^{-1} t_{b}^{-1}\right)^{-1} \text {. }
$$

Both open books that bound these fibrations contain negative Dehn twists (recall that on $-\partial X_{2}$, the monodromy is $\mu_{2}^{-1}$ ), and therefore the contact structures they support are overtwisted. As we have already manipulated the monodromy that way, contact structures and open books are isotopic, so we can glue $X_{1}$ and $X_{2}$ along the truncated pages. Putting in $S^{2} \times D^{2}$ we pass to a torus fibration $\hat{f}: Y \rightarrow S^{2}$ with global monodromy $\mu_{1} \cdot \mu_{2}$. (Applying the handle slides given in Example 4.3 repeatedly, and proceeding with the same handle cancelations, one can indeed check that $Y=\#_{8} S^{2} \times S^{2} \# \mathbb{C} \mathbb{P}^{2} \# \overline{\mathbb{C P}}^{2}$.) Now the monodromy splits easily as explained in the proof of Theorem 5.2, and we get $\mu_{+}=\left(t_{b} t_{a}\right)^{5}$ and $\mu_{-}=\left(t_{a}^{-1} t_{b}^{-1}\right)^{5}$. It is not hard to see that when we take out the section now, we get pieces $X_{+}$and $X_{-}$, which are diffeomorphic to $-E_{8}$ and $E_{8}$, respectively. So $X$ decomposes into a Stein piece $-E_{8}$ and an anti-Stein piece $E_{8}$. This defines a nicely folded Kähler structure $\omega$ on $X$, folded along the Poincaré homology sphere $\Sigma(2,3,5)$, and it is supported by a folded Lefschetz fibration which is the monodromy double of a torus fibration over $D^{2}$ minus a section.

\section{References}

[1] S Akbulut, R Matveyev, A convex decomposition theorem for 4-manifolds, Internat. Math. Res. Notices (1998) 371-381 MR1623402

[2] S Akbulut, B Ozbagci, Lefschetz fibrations on compact Stein surfaces, Geom. Topol. 5 (2001) 319-334 MR1825664

[3] D Auroux, S K Donaldson, L Katzarkov, Singular Lefschetz pencils, Geom. Topol. 9 (2005) 1043-1114 MR2140998

[4] F Bogomolov, Fillability of contact pseudoconvex manifolds, Göttingen Univ. preprint Heft 13 (1993) 1-13

[5] A Cannas da Silva, Fold-forms on four-folds, preprint (2002)

[6] A Cannas da Silva, V Guillemin, C Woodward, On the unfolding of folded symplectic structures, Math. Res. Lett. 7 (2000) 35-53 MR1748286 
[7] S K Donaldson, Lefschetz pencils on symplectic manifolds, J. Differential Geom. 53 (1999) 205-236 MR1802722

[8] Y Eliashberg, Topological characterization of Stein manifolds of dimension $>2$, Internat. J. Math. 1 (1990) 29-46 MR1044658

[9] Y Eliashberg, A few remarks about symplectic filling, Geom. Topol. 8 (2004) 277-293 MR2023279

[10] Y Eliashberg, M Gromov, Convex symplectic manifolds, from: "Several complex variables and complex geometry, Part 2 (Santa Cruz, CA, 1989)", Proc. Sympos. Pure Math. 52, Amer. Math. Soc., Providence, RI (1991) 135-162 MR1128541

[11] J B Etnyre, Symplectic convexity in low-dimensional topology, Topology Appl. 88 (1998) 3-25 MR1634561

[12] J B Etnyre, T Fuller, Realizing 4-manifolds as achiral Lefschetz fibrations, Int. Math. Res. Not. (2006) Art. ID 70272, 21 MR2219214

[13] D T Gay, Explicit concave fillings of contact three-manifolds, Math. Proc. Cambridge Philos. Soc. 133 (2002) 431-441 MR1919715

[14] E Giroux, Structures de contact en dimension trois et bifurcations des feuilletages de surfaces, Invent. Math. 141 (2000) 615-689 MR1779622

[15] E Giroux, Géométrie de contact: de la dimension trois vers les dimensions supérieures, from: "Proceedings of the International Congress of Mathematicians, Vol. II (Beijing, 2002)", Higher Ed. Press, Beijing (2002) 405-414 MR1957051

[16] RE Gompf, Sums of elliptic surfaces, J. Differential Geom. 34 (1991) 93-114 MR1114454

[17] R E Gompf, Handlebody construction of Stein surfaces, Ann. of Math. (2) 148 (1998) 619-693 MR1668563

[18] R E Gompf, A I Stipsicz, 4-manifolds and Kirby calculus, Graduate Studies in Mathematics 20, American Mathematical Society, Providence, RI (1999) MR1707327

[19] H Grauert, On Levi's problem and the imbedding of real-analytic manifolds, Ann. of Math. (2) 68 (1958) 460-472 MR0098847

[20] J L Harer, Pencils of curves of 4-manifolds, PhD thesis, University of California, Berkeley (1979)

[21] K Honda, On the classification of tight contact structures. I, Geom. Topol. 4 (2000) 309-368 MR1786111

[22] P B Kronheimer, T S Mrowka, Monopoles and contact structures, Invent. Math. 130 (1997) 209-255 MR1474156

[23] A Loi, R Piergallini, Compact Stein surfaces with boundary as branched covers of $B^{4}$, Invent. Math. 143 (2001) 325-348 MR1835390 
[24] B Ozbagci, A I Stipsicz, Contact 3-manifolds with infinitely many Stein fillings, Proc. Amer. Math. Soc. 132 (2004) 1549-1558 MR2053364

[25] B Ozbagci, A I Stipsicz, Surgery on contact 3-manifolds and Stein surfaces, Bolyai Society Mathematical Studies 13, Springer, Berlin (2004) MR2114165

[26] O Plamenevskaya, Contact structures with distinct Heegaard Floer invariants, Math. Res. Lett. 11 (2004) 547-561 MR2092907

[27] F Quinn, Dual decompositions of 4-manifolds, Trans. Amer. Math. Soc. 354 (2002) 1373-1392 MR1873010

[28] W P Thurston, Some simple examples of symplectic manifolds, Proc. Amer. Math. Soc. 55 (1976) 467-468 MR0402764

[29] W P Thurston, H E Winkelnkemper, On the existence of contact forms, Proc. Amer. Math. Soc. 52 (1975) 345-347 MR0375366

[30] J von Bergmann, Pseudo-holomorphic maps into folded symplectic four-manifolds arXiv:math.SG/0511597

Department of Mathematics, Michigan State University

East Lansing MI 48824, USA

baykur@msu .edu

Received: 13 May 2006

Algebraic $6 \mathcal{G}$ Geometric Topology, Volume 6 (2006) 